Finite pointset method based on the projection method for simulations of the incompressible Navier-Stokes equations 
(c) Fraunhofer-Institut für Techno- und

Wirtschaftsmathematik ITWM 2001

\section{ISSN 1434-9973}

Bericht 30 (2001)

Alle Rechte vorbehalten. Ohne ausdrückliche, schriftliche Genehmigung des Herausgebers ist es nicht gestattet, das Buch oder Teile daraus in irgendeiner Form durch Fotokopie, Mikrofilm oder andere Verfahren zu reproduzieren oder in eine für Maschinen, insbesondere Datenverarbeitungsanlagen, verwendbare Sprache zu übertragen. Dasselbe gilt für das Recht der öffentlichen Wiedergabe.

Warennamen werden ohne Gewährleistung der freien Verwendbarkeit benutzt.

Die Veröffentlichungen in der Berichtsreihe des Fraunhofer ITWM können bezogen werden über:

Fraunhofer-Institut für Techno- und

Wirtschaftsmathematik ITWM

Gottlieb-Daimler-Straße, Geb. 49

\section{Kaiserslautern}

Telefon: +49 (0) 6 31/2 05-32 42

Telefax: +49 (0) 6 31/2 05-4139

E-Mail: info@itwm.fhg.de

Internet: www.itwm.fhg.de 
Das Tätigkeitsfeld des Fraunhofer Instituts für Techno- und Wirtschaftsmathematik ITWM umfasst anwendungsnahe Grundlagenforschung, angewandte Forschung sowie Beratung und kundenspezifische Lösungen auf allen Gebieten, die für Techno- und Wirtschaftsmathematik bedeutsam sind.

In der Reihe »Berichte des Fraunhofer ITWM « soll die Arbeit des Instituts kontinuierlich einer interessierten Öffentlichkeit in Industrie, Wirtschaft und Wissenschaft vorgestellt werden. Durch die enge Verzahnung mit dem Fachbereich Mathematik der Universität Kaiserslautern sowie durch zahlreiche Kooperationen mit internationalen Institutionen und Hochschulen in den Bereichen Ausbildung und Forschung ist ein großes Potenzial für Forschungsberichte vorhanden. In die Berichtreihe sollen sowohl hervorragende Diplom- und Projektarbeiten und Dissertationen als auch Forschungsberichte der Institutsmitarbeiter und Institutsgäste zu aktuellen Fragen der Techno- und Wirtschaftsmathematik aufgenommen werden.

Darüberhinaus bietet die Reihe ein Forum für die Berichterstattung über die zahlreichen Kooperationsprojekte des Instituts mit Partnern aus Industrie und Wirtschaft.

Berichterstattung heißt hier Dokumentation darüber, wie aktuelle Ergebnisse aus mathematischer Forschungs- und Entwicklungsarbeit in industrielle Anwendungen und Softwareprodukte transferiert werden, und wie umgekehrt Probleme der Praxis neue interessante mathematische Fragestellungen generieren.

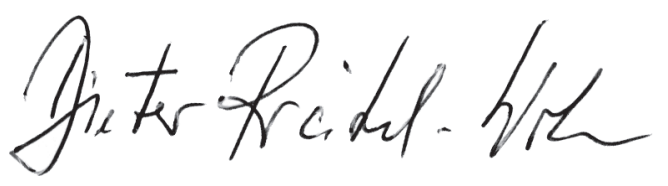

Prof. Dr. Dieter Prätzel-Wolters

Institutsleiter

Kaiserslautern, im Juni 2001 


\title{
Finite pointset method based on the projection method for simulations of the incompressible Navier-Stokes equations
}

\author{
S. Tiwari , J. Kuhnert \\ Fraunhofer Institut Techno- und Wirtschaftmathematik \\ Gottlieb-Daimler-Strasse \\ Gebäude 49 \\ D-67663 Kaiserslautern, Germany
}

Keywords: Incompressible Navier-Stokes equations, Meshfree method, Projection method, Particle scheme, Least squares approximation

AMS subject classification: 76D05, 76M28

\begin{abstract}
A Lagrangian particle scheme is applied to the projection method for the incompressible Navier-Stokes equations. The approximation of spatial derivatives is obtained by the weighted least squares method. The pressure Poisson equation is solved by a local iterative procedure with the help of the least squares method. Numerical tests are performed for two dimensional cases. The Couette flow, Poiseuelle flow, decaying shear flow and the driven cavity flow are presented. The numerical solutions are obtained for stationary as well as instationary cases and are compared with the anylatical solutions for channel flows. Finally, the driven cavity flow in a unit square is considered and the stationary solution obtained from this scheme is compared with that from the finite element method.
\end{abstract}

\section{Introduction}

The finite pointset method (FPM) is the particle method. It is a fully Lagrangian and grid free method, in which a fluid is replaced by a finite number of particles (pointset). Particles move with fluid velocities and they carry the fluid quantities, like the density, the velocity, the pressure and so on. Similarly, boundaries are also approximated by a finite number of boundary particles and boundary conditions are prescribed on them. Since it is a grid free method 
and the distribution of particles can be quite arbitrary, the method is suitable for flows with complicated geometry, free surface flows and multiphase flows. In this paper we have used a particle scheme based on the Chorin's projection method [3]

A widely used Lagrangian grid free method for solving fluid dynamic equations is the method of Smoothed Particle Hydrodynamics (SPH). The method of SPH was originally invented to solve problems in astrophysics without boundary $[7,11]$. This method has been further extended to solve varieties of problems like compressible inviscid flows, incompressible inviscid flows, multiphase flows $[4,15,12,13,14,16]$. The main difficulty of the method of SPH is the incorporation of boundary conditions.

Another approach for solving fluid dynamic equations in a grid free framework is the moving least squares or least squares method [2, 6, 5, 9, 17, 20, 18]. With this approach boundary conditions can be implemented in a natural way just by placing the particles on boundaries and prescribing boundary conditions on boundary particles [9]. The robustness of this method is shown by the simulation of airbag deployment for car industry, where the membrane (or boundary) of the airbag changes very rapidly in time and takes a quite complicated shape [10].

In the previous papers $[20,18]$ we have performed simulations of incompressible flows as the limit of the compressible Navier-Stokes equations with the quasi compressible equation of state. This approach was first used in [12] to simulate incompressible free surface flows by SPH. The incompressible limit is obtained by choosing a very large speed of sound in the equation of state such that the Mach number is of order $\approx 0.1$. However the large value of the speed of sound restricts the time step to be very small due to the CFL-condition.

The Chorin's projection method [3] is a widely used approach to solve the incompressible Navier-Stokes equation in a grid based structure. In this paper we have extended Chorin's projection method to grid free framework with the help of the weighted least squares method. The Poisson pressure equation is solved by a grid free method. In [19] it has been shown that the Poission equation can be solved accurately by this approach for any boundary conditions. The Poisson solver can be adopted in the least squares approximation procedure with the condition that the Poisson equation and the boundary condition must be satisfied on each particle. This is a local iteration procedure.

The scheme is tested for channel flows and the driven cavity flow. We have performed simulations of steady as well as unsteady flows. In the case of channel flows the numerical results are compared with the exact solutions. For the driven cavity flow the numerical solution is compared with the one obtained from the finite element method. It is found that the proposed scheme gives accurate results. 
The paper is organized as follows. In section 2, we introduce the mathematical model and the numerical scheme. In section 3, we describe the least squares method and its application for solving the pressure Poisson equation. Finally, in section 4 numerical tests are presented.

\section{Mathematical model and numerical scheme}

\subsection{Mathematical model}

We consider the incompressible Navier-Stokes equations in the Lagrangian form

$$
\begin{aligned}
& \frac{D \vec{v}}{D t}=-\frac{1}{\rho} \nabla p+\nu \Delta \vec{v}+\vec{g} \\
& \nabla \cdot \vec{v}=0,
\end{aligned}
$$

where $\rho$ is the mass density, $\vec{v}$ is the velocity vector, $\vec{g}$ is the body force acceleration vector, $\nu$ is the kinematic viscosity and $p$ the dynamic pressure. In this paper we consider a density of $\rho=1$.

The equations $(2.1-2.2)$ must be supplemented with appropriate initial and boundary conditions.

\subsection{Numerical scheme}

We consider the projection method described in [3]. This is an explicit method and is of first order accuracy in time. It consists of two fractional steps. At the first step we compute explicitely the new particle position and the intermediate velocity $\vec{v}^{*}$ which are given by

$$
\begin{aligned}
& \vec{x}^{n+1}=\vec{x}^{n}+\Delta t \vec{v}^{n} \\
& \vec{v}^{*}=\vec{v}^{n}+\Delta t \nu \Delta \vec{v}^{n}+\Delta t \vec{g}^{n} .
\end{aligned}
$$

Then, at the second step, we correct $\vec{v}^{*}$ by solving the equation

$$
\vec{v}^{n+1}=\vec{v}^{*}-\Delta t \nabla p^{n+1}
$$

with the incompressibility constraint

$$
\nabla \cdot \vec{v}^{n+1}=0
$$

By taking the divergence of equation (2.5) and by making the use of (2.6), which is the constraint that $\vec{v}^{n+1}$ must be a divergence free vector, we obtain the Poisson equation for the pressure

$$
\Delta p^{n+1}=\frac{\nabla \cdot \vec{v}^{*}}{\Delta t}
$$


The boundary condition for $p$ is obtained by projecting the equation (2.5) on the outward unit normal vector $\vec{n}$ to the boundary $\Gamma$. Thus, we obtain the Neumann boundary condition

$$
\left(\frac{\partial p}{\partial \vec{n}}\right)^{n+1}=-\frac{1}{\Delta t}\left(\vec{v}_{\Gamma}^{n+1}-\vec{v}_{\Gamma}^{*}\right) \cdot \vec{n},
$$

where $\vec{v}_{\Gamma}$ is the value of $\vec{v}$ on $\Gamma$. Assuming $\vec{v} \cdot \vec{n}=0$ on $\Gamma$, we obtain

$$
\left(\frac{\partial p}{\partial \vec{n}}\right)^{n+1}=0
$$

on $\Gamma$.

We note that the particle positions change only in the first step. The intermediate velocity $\vec{v}^{*}$ is obtained on the new particle positions. Finally, the pressure Possion equation and the divergence free velocity vector are computed also on the same new particle positions.

We approximate the spatial derivatives appearing in (2.4) and (2.5) by the weighted least squares method. Furthermore, the Possion pressure equation (2.7) is also adopted by the least squares approximation. In the following, we describe the method of approximation of spatial derivatives and the Poisson solver by the least squares method.

\section{The least squares method and the pressure Poisson solver}

\subsection{Least Squares approximation of derivatives}

The least squares method can be applied to very irregular moving geometries. In many practical applications the mesh plays a very important role in determining the solution. However, many solvers loose their accuracy if the mesh is poorly constructed. The method presented here does not require regular grids to approximate spatial derivatives of a function. This is an advantage of this method. In the begining one can distribute the particles in a regular grid but when they move with fluid velocities then their distribution becomes very irregular after short time.

In this method particle positions are itself points of some geometrical grid. To approximate derivatives of a function at each grid point means the approximation of derivatives at each particle position. Let $f(t, \vec{x})$ be a scalar function and $f_{i}(t)$ its values at $\vec{x}_{i}$ for $i=1,2, \ldots, N$ and time $t$. Consider the problem to approximate the spatial derivatives of a function $f(t, \vec{x})$ at particle position $\vec{x}$ from the values of its neighboring points. To restrict the number of points we introduce a weight function $w=w\left(\vec{x}_{i}-\vec{x} ; h\right)$ with small compact support, 
where $h$ determines the size of the support, or in other words, the length of interaction between particle.

The weight function can be quite arbitrary but in our computation, we consider a Gaussian weight function of the following form

$$
w\left(\vec{x}_{i}-\vec{x} ; h\right)= \begin{cases}\exp \left(-\alpha \frac{\left\|\vec{x}_{i}-\vec{x}\right\|^{2}}{h^{2}}\right), & \text { if } \frac{\left\|\vec{x}_{i}-\vec{x}\right\|}{h} \leq 1 \\ 0, & \text { else, }\end{cases}
$$

where $\alpha$ is a positive constant and is considered to be equal to 6.25 . The size of $h$ defines a set of neighboring particles around $\vec{x}$. Let $P(\vec{x}, h)=\left\{\vec{x}_{i}: i=\right.$ $1,2, \ldots, n\}$ be the set of $n$ neighboring points of $\vec{x}$ in a ball of radius $h$. For consistency reasons some obvious restrictions are required, for example, in $2 D$ there should be at least 5 particles and they should be neither be on the same line nor on a circle.

The determination of derivatives of a function can be computed easily and accurately by using the Taylor's series expansion and the least squares approximation. We write a Taylor expansion around the point $\vec{x}$ with unknown coefficients and then compute these coefficients by minimizing a weighted error over the neighboring points.

Consider Taylor's expansion of $f\left(t, \overrightarrow{x_{i}}\right)$ around $\vec{x}$

$$
\begin{aligned}
f\left(t, \vec{x}_{i}\right)=f( & t, \vec{x})+\sum_{k=1}^{3} f_{k}(t, \vec{x})\left(x_{k i}-x_{k}\right) \\
& +\frac{1}{2} \sum_{k, l=1}^{3} f_{k l}(t, \vec{x})\left(x_{k i}-x_{k}\right)\left(x_{l i}-x_{l}\right)+e_{i},
\end{aligned}
$$

where $e_{i}$ is the error in the Taylor series expansion at the point $\vec{x}_{i}$. The symbols $x_{1 i}, x_{2 i}$ and $x_{3 i}$ are denoted by the $x, y$ and $z$ components of the particle position $\vec{x}_{i}$ respectively. The unknowns $f_{k}$ and $f_{k l}\left(=f_{l k}\right)$ for $k, l=1,2,3$ are computed by minimizing the error $e_{i}$ for $i=1,2, \ldots, n$, where $f(t, \vec{x})=f$ is the given value of a function at the particle position $\vec{x}$. The system of equations can be written as

$$
\vec{e}=M \vec{a}-\vec{b}
$$

where $M=$

$\left(\begin{array}{ccccccccc}\Delta x 1_{1} & \Delta x 2_{1} & \Delta x 3_{1} & \Delta x 11_{1} & \Delta x 12_{1} & \Delta x 13_{1} & \Delta x 22_{1} & \Delta x 23_{1} & \Delta x 33_{1} \\ \Delta x 1_{2} & \Delta x 2_{2} & \Delta x 3_{2} & \Delta x 11_{2} & \Delta x 12_{2} & \Delta x 13_{2} & \Delta x 22_{2} & \Delta x 23_{2} & \Delta x 33_{2} \\ \vdots & \vdots & \vdots & \vdots & \vdots & \vdots & \vdots & \vdots & \vdots \\ \Delta x 1_{n} & \Delta x 2_{n} & \Delta x 3_{n} & \Delta x 11_{n} & \Delta x 12_{n} & \Delta x 13_{n} & \Delta x 22_{n} & \Delta x 23_{n} & \Delta x 33_{n}\end{array}\right)$,

where $\vec{a}=\left[f_{1}, f_{2}, f_{3}, f_{11}, f_{12}, f_{13}, f_{22}, f_{23}, f_{33}\right]^{T}, \vec{b}=\left[f_{1}-f, f_{2}-f, \ldots, f_{n}-f\right]^{T}$, $\vec{e}=\left[e_{1}, e_{2}, \ldots, e_{n}\right]^{T}$. The symbols $\Delta x k_{i}$ are defined by $x_{k i}-x_{k}$ and $\Delta x k l_{i}=$ 
$\left(x_{k i}-x_{k}\right)\left(x_{l i}-x_{l}\right)(k \neq l)$ and $\Delta x k k_{i}=\left(x_{k i}-x_{k}\right) *\left(x_{k i}-x_{k}\right) / 2$ for $k, l=1,2,3$ and $i=1,2, \ldots, n$.

For $n>9$, this system is over-determined with respect to the nine unknowns $f_{k}$ and $f_{k l}\left(=f_{l k}\right)$ for $k, l=1,2,3$.

The unknown vector $\vec{a}$ is obtained from a weighted least squares method by minimizing the quadratic form

$$
J=\sum_{i=1}^{n} w_{i} e_{i}^{2}
$$

The above equations can be expressed in the form

$$
J=(M \vec{a}-\vec{b})^{T} W(M \vec{a}-\vec{b})
$$

where

$$
W=\left(\begin{array}{cccc}
w_{1} & 0 & \cdots & 0 \\
0 & w_{2} & \cdots & 0 \\
\vdots & \vdots & \cdots & \vdots \\
0 & 0 & \cdots & w_{n}
\end{array}\right)
$$

The minimization of $J$ formally yields

$$
\vec{a}=\left(M^{T} W M\right)^{-1}\left(M^{T} W\right) \vec{b} .
$$

The Taylor series expansion may include higher order expansion and appropriate discrete weight functions can be used to force the least square approximations to recover finite difference discretization.

\subsection{A least squares approach for the pressure Poisson equation}

We want to solve the following pressure Poisson equation

$$
\Delta p=\frac{\nabla \cdot \vec{v}^{*}}{\Delta t}
$$

with the boundary condition

$$
\frac{\partial p}{\partial \vec{n}}=0
$$

Here the symbol $p$ is denoted by $p^{n+1}$ for the sake of simplicity.

Since we consider problems in a grid free structure, it is not obvious to apply classical methods like the finite differences or the finite elements for solving the Poission equation. One can construct regular finite difference or finite elements grids and solve the Poisson equation by any classical method and then interpolating the pressure on the original particle positions. It can have smearing effects and require more computational time. If the domain 
is fixed, grids can be generated once and use for all time. However, when the computational domain changes in time and takes a complex shape, the construction of grids will be extreamly difficult.

Therefore, we use a local iteration approach on the basis of the least squares approximation, where the Poisson equation is forced to satisfy on each particle position. This approach can be slower than other Poisson solvers. But the main advantage is that it does not require any reconstruction of grids and can be used for complex geometries. This methods is stable and gives accurate results for all boundary value problems of the Poisson equation, see [19] for details.

In the previous subsection we have presented the least squares method to approximate derivatives of a function at an arbitrary point from its neighbouring values. The approximation of a function at a point can be obtained from its neighbouring values by the least squares method. However, the values of pressure at the new particle positions are not known a priori. Hence, we prescribe an initial guess $p^{(0)}$ for the pressure $p$. Now, we consider the problem of determining $p$ at a particle position $\vec{x}$ from its neighbouring points $\vec{x}_{i}, i=1, \ldots, n$. As described in the previous section, we again consider a Taylor's expansion of $p$ at $\vec{x}$

$$
\begin{aligned}
p^{(\tau)}\left(\vec{x}_{i}\right)= & p^{(\tau+1)}(\vec{x})+\sum_{k=1}^{3} p_{k}^{(\tau+1)}(\vec{x})\left(x_{k i}-x_{k}\right) \\
& +\frac{1}{2} \sum_{k, l=1}^{3} p_{k l}^{(\tau+1)}(\vec{x})\left(x_{k i}-x_{k}\right)\left(x_{l i}-x_{l}\right)+e_{i}^{(\tau+1)}
\end{aligned}
$$

for $\tau=0,1,2, \ldots$, where $p^{(0)}\left(\vec{x}_{i}\right)$ is the set of given initial values. In addition, we consider the condition that the Poisson equation (3.13) must be satisfied at $\vec{x}$. Thus, we have to add the following equation in the above system of $n$ equations

$$
\frac{\nabla \cdot \vec{v}^{*}}{\Delta t}=p_{11}^{(\tau+1)}(\vec{x})+p_{22}^{(\tau+1)}(\vec{x})+p_{33}^{(\tau+1)}(\vec{x}) .
$$

Moreover, we also enforce the Neumann boundary condition (3.14) to be satisfied and incorporate one more equation if $\vec{x}$ is the position of a boundary particle

$$
0=p_{1}^{(\tau+1)}(\vec{x}) n_{x}+p_{2}^{(\tau+1)}(\vec{x}) n_{y}+p_{3}^{(\tau+1)}(\vec{x}) n_{z}
$$

where $n_{x}, n_{y}, n_{z}$ are the $x, y, z$ components of the unit normal vector $\vec{n}$ on boundary at $\vec{x}$. Hence we have a total of $n+2$ equations for 10 unknowns for boundary particles with the Neumann boundary condition.

We obtain the coefficients

$$
p^{(\tau+1)}, p_{1}^{(\tau+1)}, p_{2}^{(\tau+1)}, p_{3}^{(\tau+1)}, p_{11}^{(\tau+1)}, p_{12}^{(\tau+1)}, p_{13}^{(\tau+1)}, p_{22}^{(\tau+1)}, p_{23}^{(\tau+1)}, p_{33}^{(\tau+1)}
$$


for $\tau=1,2,3, \ldots$ at $\vec{x}$ by minimizing

$$
J=\sum_{i=1}^{n} w_{i}\left(e_{i}^{(\tau+1)}\right)^{2}+\left(\Delta p^{(\tau+1)}-\frac{\nabla \cdot \vec{v}^{*}}{\Delta t}\right)^{2}+\left(\frac{\partial p^{(\tau+1)}}{\partial \vec{n}}-0\right)^{2} .
$$

Similarly, the minimization of $J$ is given by

$$
\vec{a}^{(\tau+1)}=\left(M^{T} W M\right)^{-1}\left(M^{T} W\right) \vec{b}^{(\tau)}, \tau=0,1, \ldots,
$$

where the matrices and the vectors differ from (3.12) and are given by $M=$

$$
\begin{gathered}
\left(\begin{array}{cccccccccc}
1 & \Delta x 1_{1} & \Delta x 2_{1} & \Delta x 3_{1} & \Delta x 11_{1} & \Delta x 12_{1} & \Delta x 13_{1} & \Delta x 22_{1} & \Delta x 23_{1} & \Delta x 33_{1} \\
1 & \Delta x 1_{2} & \Delta x 2_{2} & \Delta x 3_{2} & \Delta x 11_{2} & \Delta x 12_{2} & \Delta x 13_{2} & \Delta x 22_{2} & \Delta x 23_{2} & \Delta x 33_{2} \\
1 & \vdots & \vdots & \vdots & \vdots & \vdots & \vdots & \vdots & \vdots & \vdots \\
1 & \Delta x 1_{n} & \Delta x 2_{n} & \Delta x 3_{n} & \Delta x 11_{n} & \Delta x 12_{n} & \Delta x 13_{n} & \Delta x 22_{n} & \Delta x 23_{n} & \Delta x 33_{n} \\
0 & 0 & 0 & 0 & 1 & 0 & 0 & 1 & 0 & 1 \\
0 & n_{x} & n_{y} & n_{z} & 0 & 0 & 0 & 0 & 0 & 0
\end{array}\right), \\
W=\left(\begin{array}{cccccc}
w_{1} & 0 & \cdots & 0 & 0 & 0 \\
0 & w_{2} & \cdots & 0 & 0 & 0 \\
\vdots & \vdots & \cdots & \vdots & \vdots & \vdots \\
0 & 0 & \cdots & w_{n} & 0 & 0 \\
0 & 0 & \cdots & 0 & 1 & 0 \\
0 & 0 & \cdots & 0 & 0 & 1
\end{array}\right), \\
\vec{a}^{(\tau+1)}=\left[p^{(\tau+1)}, p_{1}^{(\tau+1)}, p_{2}^{(\tau+1)}, p_{3}^{(\tau+1)}, p_{11}^{(\tau+1)}, p_{12}^{(\tau+1)}, p_{13}^{(\tau+1)}, p_{22}^{(\tau+1)}, p_{23}^{(\tau+1)}, p_{33}^{(\tau+1)}\right]^{T}
\end{gathered}
$$

The matrices $M$ and $W$ and the vector $\vec{b}^{(\tau)}$ given above are for boundary particles. For interior particles the last row of the matrices $M$ and $W$ and the last component of the vector $\vec{b}^{(\tau)}$ are omitted.

Iterations are performed for each particle. Note that, from the vector $\vec{a}^{(\tau+1)}$, only the values $p^{(\tau+1)}$ are used for the next iteration cycle. All other values are forgotten. This is a local solution procedure. For each particle, for example, in $3 D$-case one has to invert a $10 \times 10$ matrix.

The iteration is stopped if the error satisfies

$$
\frac{\sum_{i=1}^{N}\left|p_{i}^{(\tau+1)}-p_{i}^{(\tau)}\right|}{\sum_{i=1}^{N}\left|p_{i}^{(\tau+1)}\right|}<\epsilon
$$

and the solution is defined by $p\left(x_{i}\right):=p^{(\tau+1)}\left(x_{i}\right)$ as $\tau$ tends to infinity. The parameter $\epsilon$ is a small positive constant. 
It is also necessary to prescribe the initial value of the pressure at time $t=0$. In the pressure iteration the initial guess of the pressure for the time level $n+1$ is taken as the pressure from the time level $n$. If the pressure at the old time step is close to that of new time step a few number of iterations is required.

\subsection{Restriction on time step}

Since the scheme is explicit, some restrictions are necessary for the stability. It is required that the time step satisfy

$$
\Delta t \leq \min \left\{0.15 \frac{h}{U_{\max }}, 0.1531 \frac{h^{2}}{\nu}, 0.04 \sqrt{\frac{h}{|\vec{g}|}}\right\} .
$$

\section{Numerical Tests}

We have tested different kinds of channel flow in stationary as well as instationary cases and have compared the numerical solutions with the exact ones. Both solutions match accurately.

Finally, the driven Cavity flow for different Renolds numbers is considered. We have compared the particle solutions with the finite element solutions in cases of stationary and found a very good agreement of both solutions.

\subsection{Couette flow}

The first test case is the Couette flow between infinite plates. The initial flow is at rest and the plates are located at $y=0$ and at $y=L$. At time $t=0$ the upper plate moves at constant velocity $U_{0}$ parallel to the x-axis.

The computational domain is considered as $[0, K] \times[0, L]$. The initial conditions are

$$
u(x, y, 0)=v(x, y, 0)=0, \quad p(x, y, 0)=p_{0}=0 .
$$

The Dirichlet boundary conditions on the two plates and the periodic boundary conditions on the inflow and outflow of the channel are considered. More explicitely,

$$
\begin{array}{r}
u(x, 0, t)=v(x, 0, t)=0, \quad u(x, L, t)=U_{0}, \quad v(x, L, 0)=0 \\
u(0, y, t)=u(K, y, t), \quad v(0, y, t)=v(K, y, t) .
\end{array}
$$

In order to apply periodic boundary conditions we make pairs of inflow and outflow boundary particles having the same $y$ coordinates. Then the value on the left boundary particles is assigned by the value of its pair on the right boundary. 


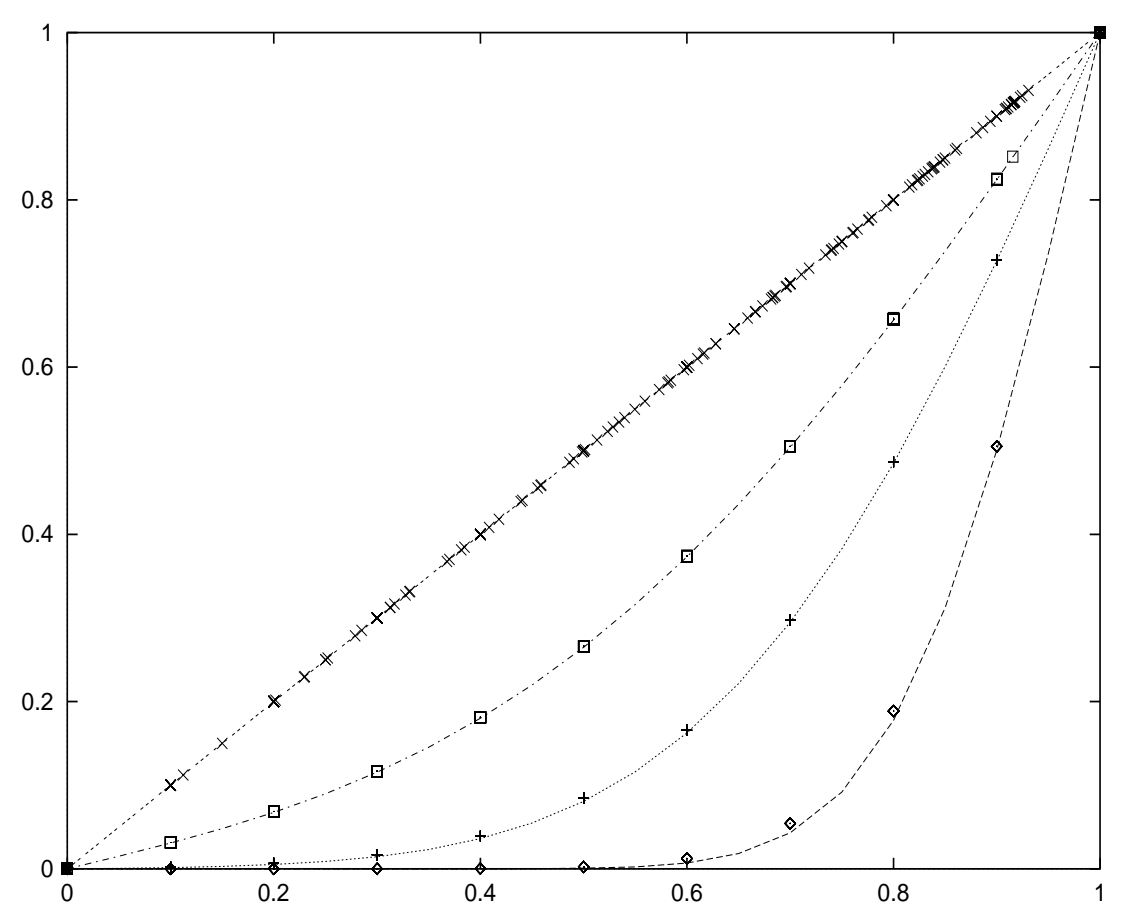

FIG. 1. Velocity component $u$ at time $0.011,0.041,0.101$ and $\infty$ from right to left respectively. Solid lines are the anylytical solutions and the stars are the numerical solutions

For the pressure Poisson equation, the Neumann boundary condition $\frac{\partial p}{\partial \vec{n}}=$ 0 is used only on the top and the bottom of the plates.

For this simple flow the analytical solution is available and the resulting steady flow is linear with a slope equal to $U_{0} y / L$. We test the steady and unsteady flow $\vec{v}=(u, v)$ against the following exact solution

$$
\begin{aligned}
& u(\vec{x}, t)=\frac{U_{0}}{L} y+\frac{2 U_{0}}{\pi} \sum_{n=1}^{\infty} \frac{(-1)^{n}}{n} \sin \left(\frac{n \pi}{L} y\right) \exp \left(-\frac{n^{2} \pi^{2}}{L^{2}} \nu t\right) \\
& v(\vec{x}, t)=0
\end{aligned}
$$

Figure 1 shows a comparison between the exact solution and the particle solution obtained for $K=2, L=1, U_{0}=1$ and $\nu=1$. The numerical solutions with points and the analytical solutions with lines at times $t=0.011, t=0.041$, $t=0.101$ and $t=\infty$, from right to left, respectively, are plotted. There is a close agreement between the exact solution and the numerical solution.

In the begining the particles are replaced in a lattice with a uniform spacing of size $\Delta x=0.1$ and the size of the support $h$ of the weight function is considered 2.8 times $\Delta x$.

For the stability some adaptivity is used. If there is a hole in the fluid domain, then a new particle is introduced at that position and the fluid values 
on this new particles are given by the least squares approximation. Further, if two particles are very close each other, one of them is removed in order to reduce the computational time. If particles leave the computational domain, they are removed.

\subsection{Poisseuelle flow}

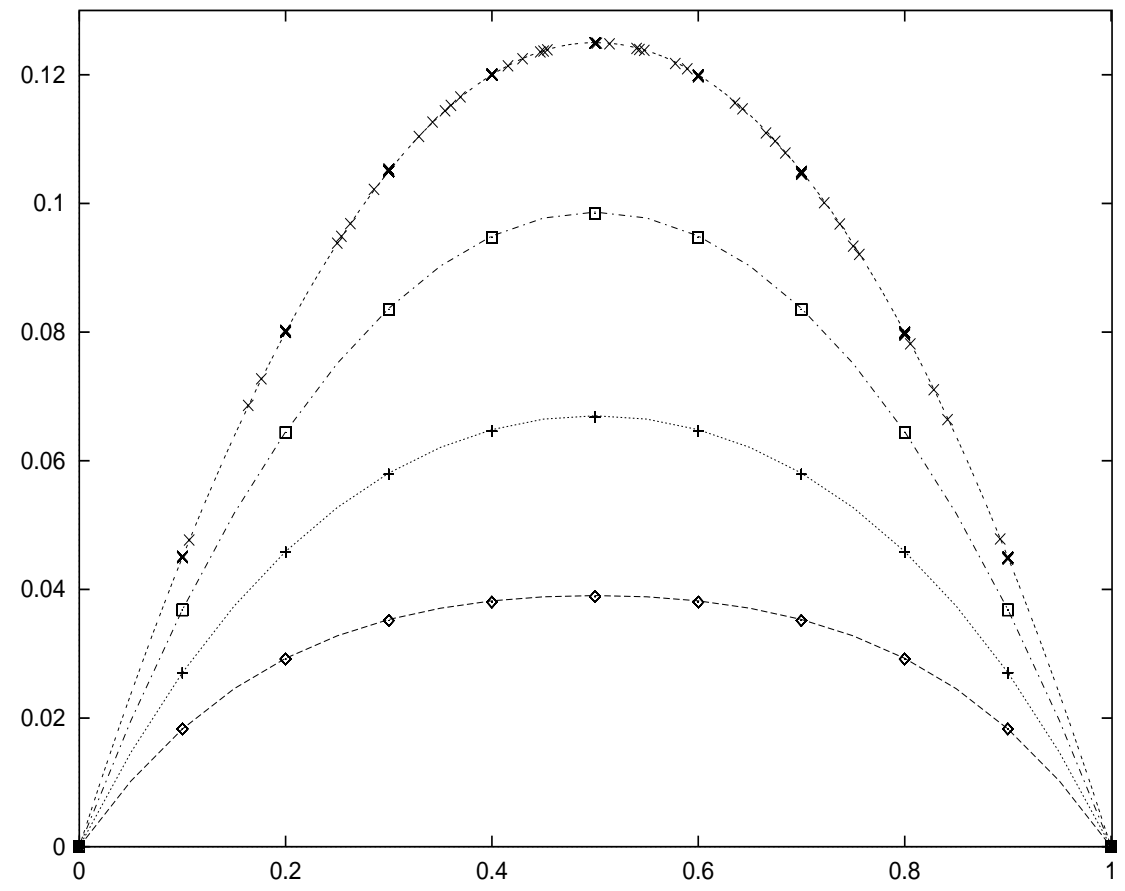

FIG. 2. Velocity $u$ at time $0.041,0.081,0.161$ and $\infty$ from bottom to top respectively. Solid lines are the anylytical solutions and the stars are the numerical solutions

The second test case is a stationary forced flow through a channel between two infinite parallel plates. The computational domain is again, considered to be a rectangle of size $[0, K] \times[0, L]$. The solution $\vec{v}=(u, v)$ of this simple flow can be written in series form as

$$
\begin{aligned}
& u(\vec{x}, t)=\frac{g_{x}}{2 \nu} y(y-L) \\
& +\frac{4 g_{x} L^{2}}{\nu \pi^{3}} \sum_{n=0}^{\infty} \frac{1}{(2 n+1)^{3}} \sin \left(\frac{\pi y}{L}(2 n+1)\right) \exp \left(-\nu \frac{(2 n+1)^{2} \pi^{2}}{L^{2}} t\right), \\
& v(\vec{x}, t)=0,
\end{aligned}
$$

where $\vec{g}=\left(g_{x}, 0\right)$ is the force. The test was performed with $K=2, L=1$, $g_{x}=1$ and $\nu=1$. 
The periodic boundary conditions in the inflow and outflow of the channel and the homogeneous Dirichlet boundary conditions $u=v=0$ on $y=0$ and $y=1$ are used. The Neumann boundary condition is used on $y=0$ and $y=1$ for the pressure Poission equation.

Again, adaptivity is used for removing and adding particles as it was done for example of Couette flow. In Figure 2 we plot the analytical and numerical solutions over particle positions at times $t=0.041, t=0.081, t=0.161$ and $t=\infty$, from bottom to top, respectively.

Next, we consider a steady Poiseuelle flow with pressure boundary conditions. This problem was proposed in [8]. The computational domain is the rectangle $[0,2] \times[0,1]$. The initial and boundary conditions are

$$
\begin{array}{r}
u(x, y, 0)=v(x, y, 0)=0, \quad p(x, y, 0)=p_{0} ; \\
u(x, 0, t)=u(x, 1, t)=v(x, 0, t)=v(x, 1, t)=0 ; \\
p(0, y, t)=p_{\text {in }} ; \quad p(2, y, t)=p_{\text {out }},
\end{array}
$$

where $p_{0}=0.5\left(p_{\text {in }}+p_{\text {out }}\right)$, and the pressure maintained at the entrance and the exit, respectively. The Poiseuelle flow has an analytical solution

$$
\begin{aligned}
& u(x, y)=\frac{p_{\text {in }}-p_{\text {out }}}{4 \nu} y(1-y) \\
& v(x, y)=0 \\
& p(x, y)=p_{\text {in }}-\frac{p_{\text {in }}-p_{\text {out }}}{2} x .
\end{aligned}
$$

The parameters used in the simulations are $\nu=1.0, p_{\text {in }}=1.1, p_{\text {out }}=1.0$. Similarly, the uniform particle distribution with spacing size $\Delta x=0.1$ is considered in the begining. The value of $h$ is taken as 0.28 , which is 2.8 times the initial particle space. Removing and adding particles are also obtained during the simulation. In Figure 3, the analytical solutions (4.25) are plotted against the numerical solutions. The numerical and anylytical solutions are found to be in very good agreement.

\subsection{Unsteady decaying shear flow}

This test case was also proposed in [8]. The unsteady decaying flow has an analytical solution

$$
\begin{array}{r}
u(x, y, t)=A \\
v(x, y, t)=B \cos (k x-k A t) e^{-k^{2} \nu t} \\
p(x, y, t)=p(t),
\end{array}
$$

where the constants $A, B, k, \nu$, and $p(t)$ are chosen as $A=B=k=\nu=1.0$, $p(t)=p_{0}+0.01 * \sin (20 \pi t)$, and $p_{0}=3.0$ is the average pressure. The computational domain is the rectangle $[0,2 \pi] \times[0,0.16 \pi]$. The initial and boundary conditions are implemented according to the analytical solution given by 
FPM for incompressible Navier-Stokes
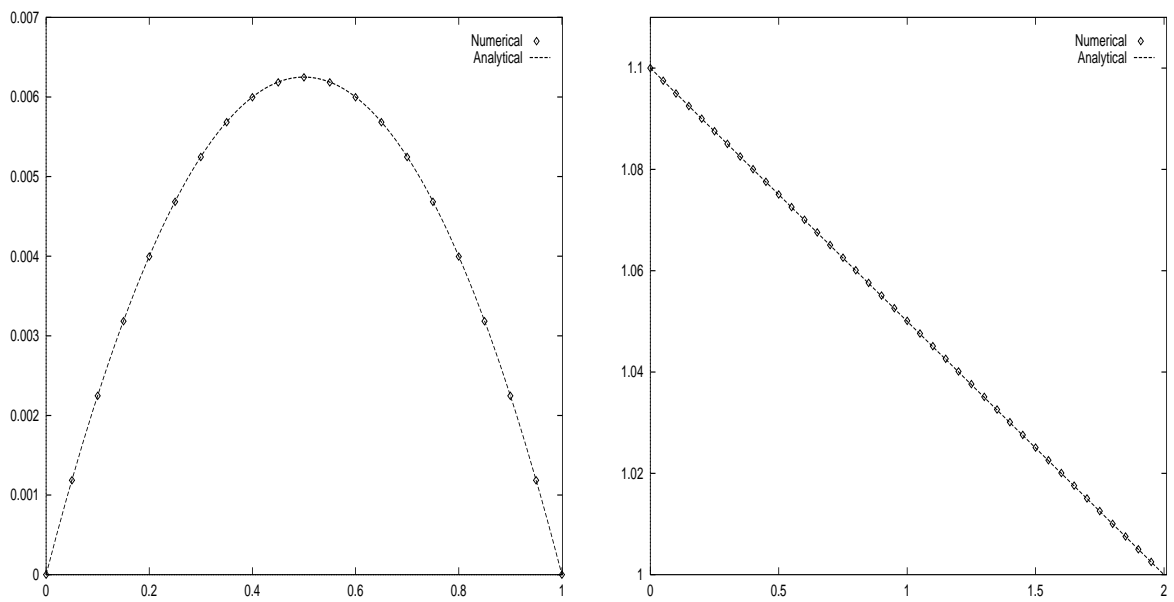

FIG. 3. Velocity u (left) and pressure $p$ (right) in steady stete

(4.25). The solutions are plotted in Figure 4 for different times where the numerical solutions match perfectly with the analytical solutions.

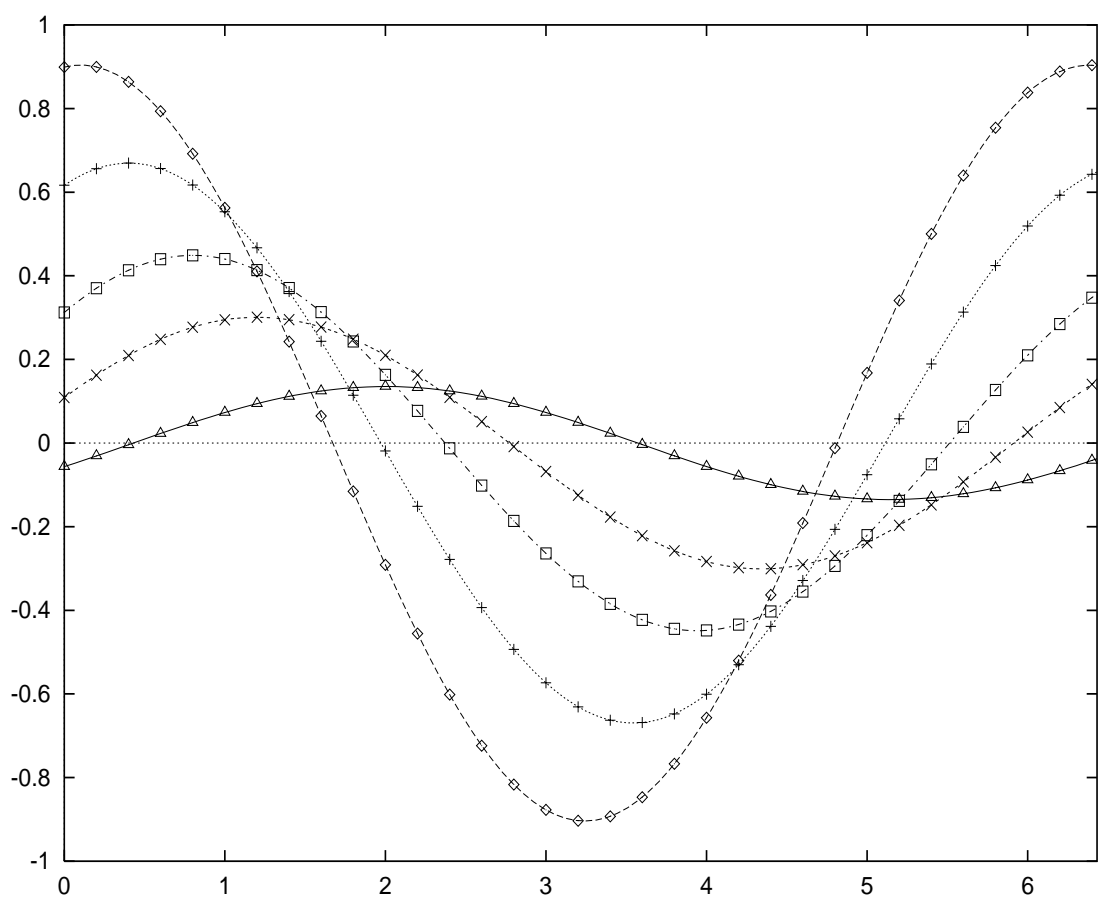

FIG. 4. Velocity $v$ at time $0.101,0.401,0.801,1.201$ and 2.001 from top to buttom, respectively. Solid lines are the anylytical solutions and the stars are the numerical solutions 


\subsection{Driven cavity flow}

The flow in a cavity driven by the velocity on the top is a standard simulation and for several years, steady flow in a square has become a popular example for testing and comparing numerical methods. In literature most of the tests are performed with zero fluid velocity on the three sides of the square and with constant tangential velocity over the fourth side. Because of the discontinuity of the boundary velocity at the corner, the solution of the Navier-Stokes equations is in some way "singular" at these points (the vorticity becomes infinite). As a consequence, it is difficult to compare solutions since the approximation of the flow close to the singularity affect the accuracy of the solution. This is particularly true when the mesh is refined so that computational points are drawn near to the corners.

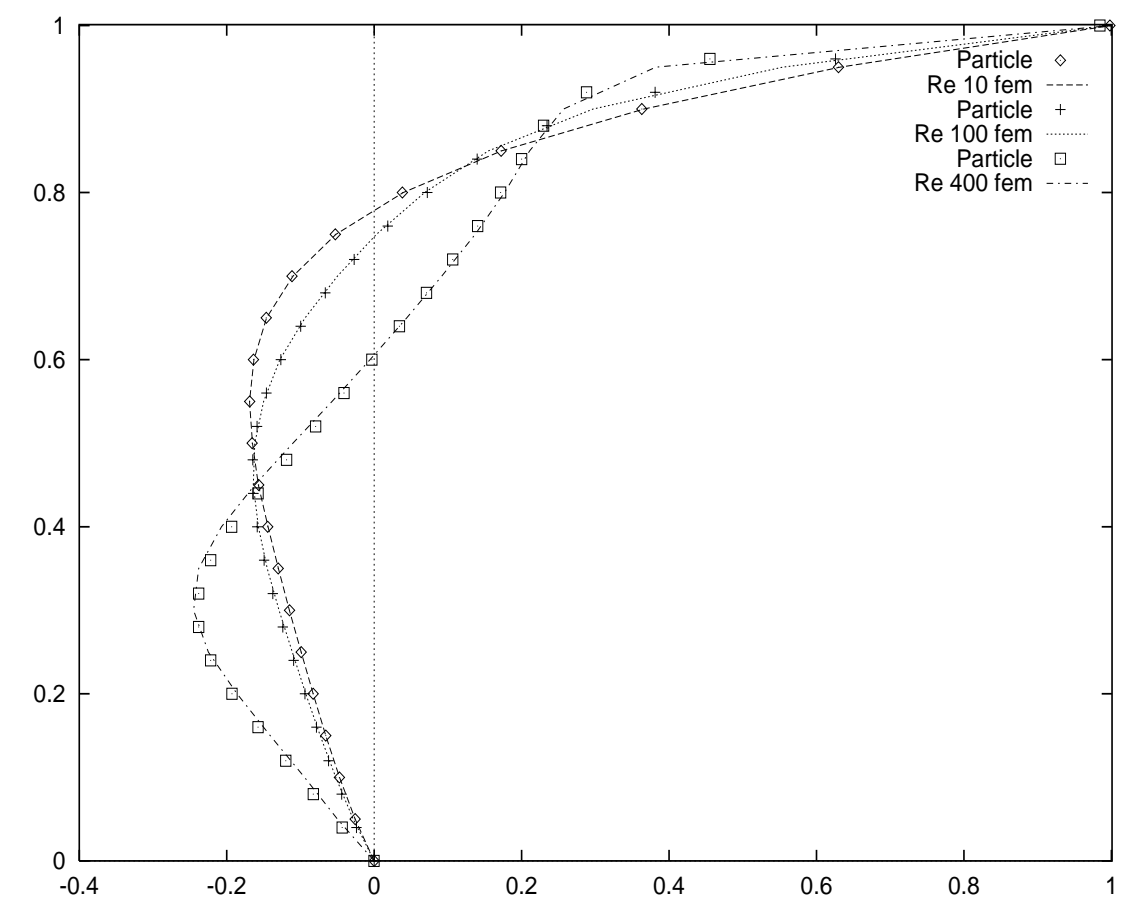

FIG. 5. Velocity $u$ of particle method with points and FEM with lines for Renold numbers 10,100 and 400

Since we are trying to compare solutions with coarse mesh we avoid such a singularity and consider a regular solution of the Navier-Stokes equations which is the solution of the cavity flow [1]. The $x$-component of the velocity $\vec{v}$ on the top side of the cavity is not longer constant but defined as

$$
u(x, 1)=16 x^{2}(1-x)^{2}, \quad v(x, 1)=0
$$



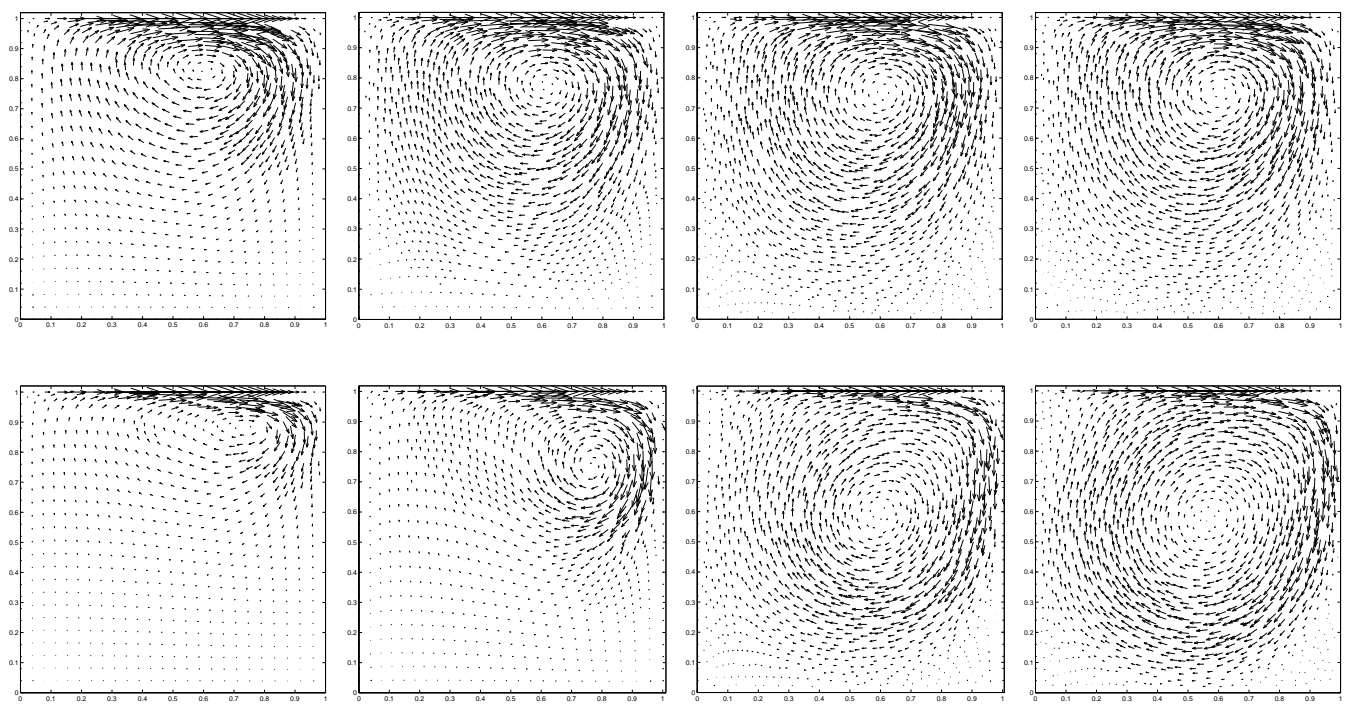

FIG. 6. Velocity profile for $R e=100$ (top) and $R e=1000$ (bottom) at time $t=1.01, t=3.01, t=10.01$ and $t=16.01$ from left to right.

and homogeneous Dirichlet boundary conditions $u=v=0$ over the rest of the boundaries. Numerical solutions are compared with finite element numerical solutions with some Reynolds numbers.

Comparison of stationary solutions with finite element solutions for Reynolds numbers equal to 10,100 and 400 is shown in Figure 5. This shows the particle solutions and finite elements solutions give same results.

The evolution of the flow field for different Reynolds numbers is different. In Figure 6 the time evolutions of the velocity field for the Renolds numbers 100 and 1000 is plotted. The flow reaches in steady state faster for a smaller Reynolds number. For smaller Reynolds number, the vortex build up in the upper center, but for the larger one it build up in the upper right corner and shifted to the center diagonally during the evolution. This phenomennon can be clearly observed in Fig. 6 .

Finally, the stationary solutions for Renolds numbers 10, 100, 400, 1000 and 5000 is plotted in Figure 7. The location of vortex changes with the Reynolds number.

\section{Conclusions}

The results of the computations show that the Lagrangian particle scheme based on the projection method gives accurate solutions of incompressible flows. The Navier-Stokes equations can be simulated with good accuracy and over a quite arbitrary distribution of particles. The pressure Poission equation 

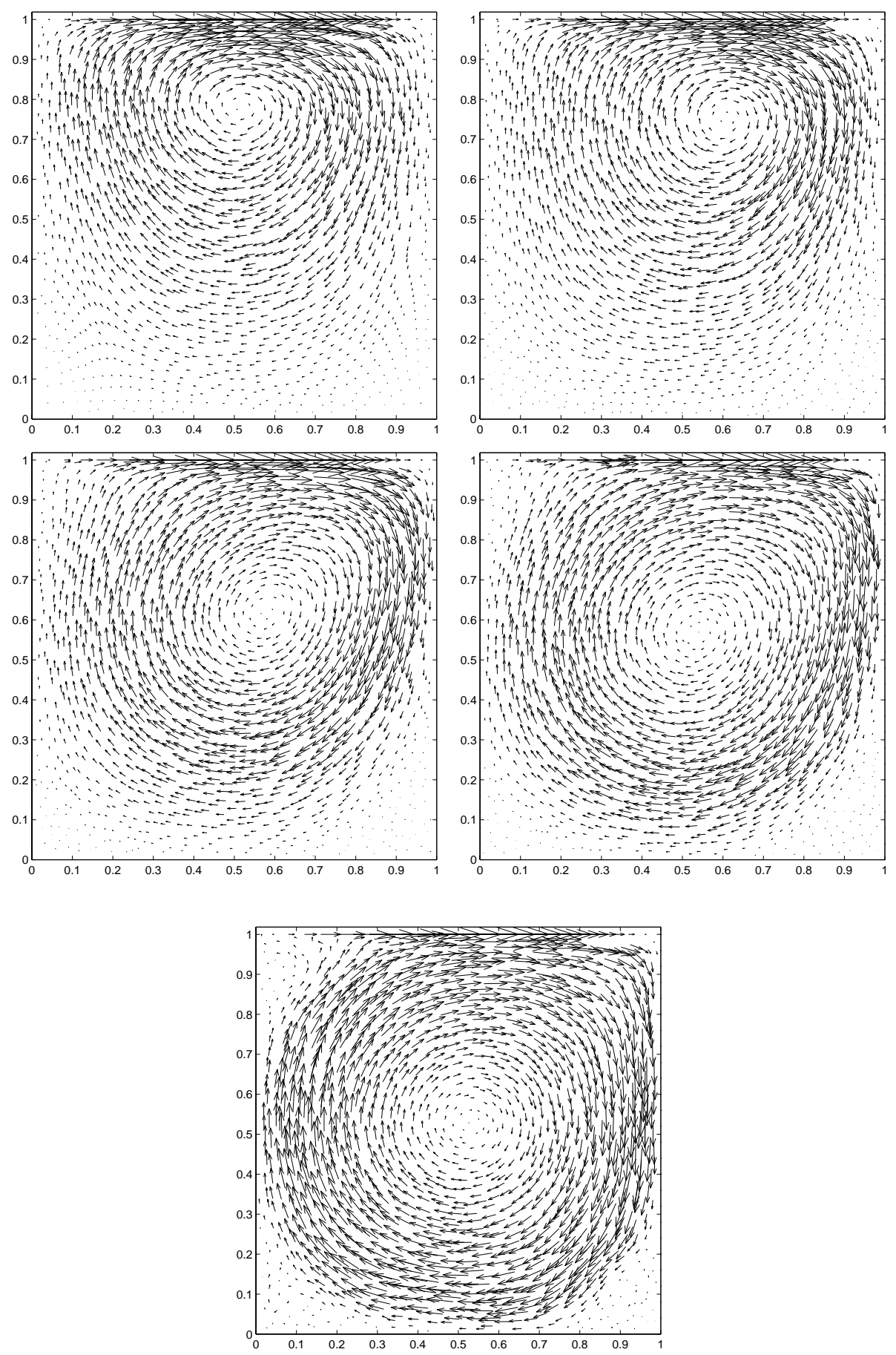

FIG. 7. Velocity profile for $R e=10$ (top left), 100 (top right), 400 (middle left), 1000 (middle right) and $R e=5000$ (bottom). 
can be solved by least squares approximation. The use of the multigrid method for solving Poisson equation is supposed to be of advantage. The future work will be the extension of this scheme for simulations of free surface flows and multiphase flows.

Acknowledgment: We thank Deutsche Forschungsgemeinschaft (DFG)- Priority Research Program: Analysis and Numerics for Conservation Laws for the financial support.

\section{References}

[1] M. Bourcier And C. Francois, C. Rech. Aèrosp. No 131, 23-33, (1969)

[2] T. Belytschko, Y. Krongauz, M. Flemming, D. Organ, W. K. S. LiU, Smoothing and accelerated computations in the element free Galerkin method; J. Comp. Appl. Maths. 74, 111-126, (1996)

[3] A. Chorin, Numerical solution of the Navier-Stokes equations; J. Math. Comput. 22 745-762 (1968)

[4] S. J. Cummins and M. Rudman, An SPH Projection Method; J. Comp. Phys. 152, 584-607 (1999)

[5] G.A DiLTs, Moving least squares particle hydrodynamics I, consistency and stability; Hydrodynamics methods group report, Los Alamos National Laboratory (1996)

[6] S.M. Deshpande And P.S. Kulkarni, New developments in kinetics schemes; Comp. Math. Appl., 35, 1 75-93, (1998)

[7] R. A. Gingold and J. J. Monaghan, Smoothed particle hydrodynamics: theory and application to non-spherical stars; Mon. Not. Roy. Astron. Soc. 181, 375-389, (1977)

[8] Z. Guo, B. SHI, N. WANG, Lattice BGK Model for incompressible Navier-Stokes equations, J. Comp. Phys., 165 288-306 (2000)

[9] J. Kuhnert, General smoothed particle hydrodynamics; Ph.D. thesis, Kaiserslautern University, Germany (1999)

[10] J. Kuhnert, A. Tramecon, P. Ullrich, Advanced Air Bag Fluid Structure Coupled Simulations applied to out-of Position Cases; EUROPAM Conference Proceedings 2000, ESI group, Paris, France

[11] L. B. LuCY, A numerical approach to the testing of the fission hypothesis; Astron. J., 82, 1013, (1977)

[12] J.J.Monaghan, Smoothed particle hydrodynamics; Annu. Rev. Astron. Astrop, 30, 543-574, (1992) 
[13] J.J.Monaghan, Simulating free surface flows with SPH; J. Comput. Phys., 110, 399, (1994)

[14] J. J. Monaghan, A. Kocharyan, SPH simulation of multi-phase flow; Computer Phys. Commun., 87, 225-235, (1995)

[15] J. J. Monaghan And R. A: Gingold, Shock Simulation by particle method SPH; J. Comp. Phys., 52, 374-389, (1983)

[16] J.P.Morris, P.J.Fox AND Y.Zhu, Modeling Low Reynolds Number Incompressible Flows Using SPH; J. Comput. Phys., 136, 214-226, (1997)

[17] S. TIWARI, A LSQ-SPH approach for compressible viscous flows, to appear in Proceedings of the 8th International Conference on Hyperbolic Problems Hyp2000

[18] S. Tiwari, J. Kunnert, Particle method for simulations of free surface flows, (submitted)

[19] S. Tiwari, J. Kuhnert, Grid free method for solving Poisson equation, preprint, Berichte des Fraunhofer ITWM, Kaiserslautern, Germany, Nr. 25 (2001)

[20] S. TIWARI AND S. MANSERVISI Modeling incompressible Navier-Stokes flows by $L S Q-S P H$, preprint, ITWM, Germany (2000) 


\author{
Die PDF-Files der folgenden Berichte \\ finden Sie unter: \\ www.itwm.fhg.de/zentral/berichte.html
}

1. D. Hietel, K. Steiner, J. Struckmeier

\section{A Finite - Volume Particle Method for} Compressible Flows

We derive a new class of particle methods for conservation laws, which are based on numerical flux functions to model the interactions between moving particles. The derivation is similar to that of classical Finite-Volume methods; except that the fixed grid structure in the Finite-Volume method is substituted by so-called mass packets of particles. We give some numerical results on a shock wave solution for Burgers equation as well as the well-known one-dimensional shock tube problem. (19 S., 1998)

\section{M. Feldmann, S. Seibold}

\section{Damage Diagnosis of Rotors: Application of Hilbert Transform and Multi-Hypothesis Testing}

In this paper, a combined approach to damage diagnosis of rotors is proposed. The intention is to employ signalbased as well as model-based procedures for an improved detection of size and location of the damage. In a first step, Hilbert transform signal processing techniques allow for a computation of the signal envelope and the instantaneous frequency, so that various types of nonlinearities due to a damage may be identified and classified based on measured response data. In a second step a multi-hypothesis bank of Kalman Filters is employed for the detection of the size and location of the damage based on the information of the type of damage provided by the results of the Hilbert transform.

Keywords:

Hilbert transform, damage diagnosis, Kalman filtering, non-linear dynamics

(23 S., 1998)

\section{Y. Ben-Haim, S. Seibold}

\section{Robust Reliability of Diagnostic Multi- Hypothesis Algorithms: Application to Rotating Machinery}

Damage diagnosis based on a bank of Kalman filters, each one conditioned on a specific hypothesized system condition, is a well recognized and powerful diagnostic tool. This multi-hypothesis approach can be applied to a wide range of damage conditions. In this paper, we will focus on the diagnosis of cracks in rotating machinery. The question we address is: how to optimize the multihypothesis algorithm with respect to the uncertainty of the spatial form and location of cracks and their resulting dynamic effects. First, we formulate a measure of the reliability of the diagnostic algorithm, and then we discuss modifications of the diagnostic algorithm for the maximization of the reliability. The reliability of a diagnos tic algorithm is measured by the amount of uncertainty consistent with no-failure of the diagnosis. Uncertainty is quantitatively represented with convex models. Keywords:

Robust reliability, convex models, Kalman filtering, multihypothesis diagnosis, rotating machinery, crack diagnosis (24 S., 1998)

\section{F.-Th. Lentes, N. Siedow}

Three-dimensional Radiative Heat Transfer in Glass Cooling Processes

For the numerical simulation of 3D radiative heat transfer in glasses and glass melts, practically applicable mathematical methods are needed to handle such problems optimal using workstation class computers. Since the exact solution would require super-computer capabilities we concentrate on approximate solutions with a high degree of accuracy. The following approaches are studied: 3D diffusion approximations and 3D ray-tracing methods

(23 S., 1998)

\section{A. Klar, R. Wegener}

\section{A hierarchy of models for multilane vehicular traffic Part I: Modeling}

In the present paper multilane models for vehicular traffic are considered. A microscopic multilane model based on reaction thresholds is developed. Based on this model an Enskog like kinetic model is developed. In particular, care is taken to incorporate the correlations between the vehicles. From the kinetic model a fluid dynamic model is derived. The macroscopic coefficients are deduced from the underlying kinetic model. Numerical simulations are presented for all three levels of description in [10]. Moreover, a comparison of the results is given there.

(23 S., 1998)

\section{Part II: Numerical and stochastic investigations}

In this paper the work presented in [6] is continued. The present paper contains detailed numerical investigations of the models developed there. A numerical method to treat the kinetic equations obtained in [6] are presented and results of the simulations are shown. Moreover, the stochastic correlation model used in [6] is described and investigated in more detail.

(17 S., 1998)

\section{A. Klar, N. Siedow}

Boundary Layers and Domain Decomposition for Radiative Heat Transfer and Diffusion Equations: Applications to Glass Manufacturing Processes

In this paper domain decomposition methods for radiative transfer problems including conductive heat transfe are treated. The paper focuses on semi-transparent materials, like glass, and the associated conditions at the interface between the materials. Using asymptotic analysis we derive conditions for the coupling of the radiative transfer equations and a diffusion approximation. Several test cases are treated and a problem appearing in glass manufacturing processes is computed. The results clearly show the advantages of a domain decomposition approach. Accuracy equivalent to the solution of the global radiative transfer solution is achieved, whereas computation time is strongly reduced.

(24 S., 1998)

\section{I. Choquet}

Heterogeneous catalysis modelling and numerical simulation in rarified gas flows Part I: Coverage locally at equilibrium

A new approach is proposed to model and simulate numerically heterogeneous catalysis in rarefied gas flows. It is developed to satisfy all together the following points: 1) describe the gas phase at the microscopic scale, as required in rarefied flows,

2) describe the wall at the macroscopic scale, to avoid prohibitive computational costs and consider not only crystalline but also amorphous surfaces,

3) reproduce on average macroscopic laws correlated with experimental results and

4) derive analytic models in a systematic and exact way. The problem is stated in the general framework of a non static flow in the vicinity of a catalytic and non porous surface (without aging). It is shown that the exact and systematic resolution method based on the Laplace transform, introduced previously by the author to model collisions in the gas phase, can be extended to the present problem. The proposed approach is applied to the modelling of the Eley-Rideal and Langmuir-Hinshelwood recombinations, assuming that the coverage is locally at equilibrium. The models are developed considering one atomic species and extended to the general case of several atomic species. Numerical calculations show that the models derived in this way reproduce with accuracy behaviors observed experimentally.

(24 S., 1998)

\section{J. Ohser, B. Steinbach, C. Lang}

\section{Efficient Texture Analysis of Binary Images}

A new method of determining some characteristics of binary images is proposed based on a special linear filtering. This technique enables the estimation of the area fraction, the specific line length, and the specific integral of curvature. Furthermore, the specific length of the tota projection is obtained, which gives detailed information about the texture of the image. The influence of lateral and directional resolution depending on the size of the applied filter mask is discussed in detail. The technique includes a method of increasing directional resolution for texture analysis while keeping lateral resolution as high as possible.

(17 S., 1998)

\section{J. Orlik}

\section{Homogenization for viscoelasticity of the} integral type with aging and shrinkage

A multi-phase composite with periodic distributed inclusions with a smooth boundary is considered in this contribution. The composite component materials are supposed to be linear viscoelastic and aging (of the non-convolution integral type, for which the Laplace transform with respect to time is not effectively applicable) and are subjected to isotropic shrinkage. The free shrinkage deformation can be considered as a fictitious temperature deformation in the behavior law. The procedure presented in this paper proposes a way to determine average (effective homogenized) viscoelastic and shrinkage (temperature) composite properties and the homogenized stress-field from known properties of the 
components. This is done by the extension of the asymptotic homogenization technique known for pure elastic non-homogeneous bodies to the non-homogeneous thermo-viscoelasticity of the integral non-convolution type. Up to now, the homogenization theory has not covered viscoelasticity of the integral type.

Sanchez-Palencia (1980), Francfort \& Suquet (1987) (see [2], [9]) have considered homogenization for viscoelasticity of the differential form and only up to the first derivative order. The integral-modeled viscoelasticity is more general then the differential one and includes almost all known differential models. The homogenization procedure is based on the construction of an asymptotic solution with respect to a period of the composite structure. This reduces the original problem to some auxiliary boundary value problems of elasticity and viscoelasticity on the unit periodic cell, of the same type as the original non-homogeneous problem. The existence and uniqueness results for such problems were obtained for kernels satisfying some constrain conditions. This is done by the extension of the Volterra integral operator theory to the Volterra operators with respect to the time, whose 1 kernels are space linear operators for any fixed time variables. Some ideas of such approach were proposed in [11] and [12], where the Volterra operators with kernels depending additionally on parameter were considered This manuscript delivers results of the same nature for the case of the space-operator kernels. (20 S., 1998)

\section{J. Mohring}

\section{Helmholtz Resonators with Large Aperture}

The lowest resonant frequency of a cavity resonator is usually approximated by the classical Helmholtz formula. However, if the opening is rather large and the front wal is narrow this formula is no longer valid. Here we present a correction which is of third order in the ratio of the diameters of aperture and cavity. In addition to the high accuracy it allows to estimate the damping due to radiation. The result is found by applying the method of matched asymptotic expansions. The correction contains form factors describing the shapes of opening and cavity. They are computed for a number of standard geometries. Results are compared with numerical computations. (21 S., 1998)

\section{H. W. Hamacher, A. Schöbel \\ On Center Cycles in Grid Graphs}

Finding "good" cycles in graphs is a problem of great interest in graph theory as well as in locational analysis. We show that the center and median problems are NP hard in general graphs. This result holds both for the variable cardinality case (i.e. all cycles of the graph are considered) and the fixed cardinality case (i.e. only cycles with a given cardinality $p$ are feasible). Hence it is of interest to investigate special cases where the problem is solvable in polynomial time.

In grid graphs, the variable cardinality case is, for instance, trivially solvable if the shape of the cycle can be chosen freely.

If the shape is fixed to be a rectangle one can analyze rectangles in grid graphs with, in sequence, fixed dimension, fixed cardinality, and variable cardinality. In all cases a complete characterization of the optimal cycles and closed form expressions of the optimal objective values are given, yielding polynomial time algorithms for all cases of center rectangle problems.

Finally, it is shown that center cycles can be chosen as rectangles for small cardinalities such that the center cycle problem in grid graphs is in these cases completely solved

(15 S., 1998)

12. H. W. Hamacher, K.-H. Küfer

\section{Inverse radiation therapy planning -} a multiple objective optimisation approach

For some decades radiation therapy has been proved successful in cancer treatment. It is the major task of clinical radiation treatment planning to realize on the one hand a high level dose of radiation in the cancer tissue in order to obtain maximum tumor control. On the other hand it is obvious that it is absolutely necessary to keep in the tissue outside the tumor, particularly in organs at risk, the unavoidable radiation as low as possible. No doubt, these two objectives of treatment planning high level dose in the tumor, low radiation outside the tumor - have a basically contradictory nature. Therefore, it is no surprise that inverse mathematical models with dose distribution bounds tend to be infeasible in most cases. Thus, there is need for approximations compromis ing between overdosing the organs at risk and underdosing the target volume.

Differing from the currently used time consuming iterative approach, which measures deviation from an ideal (non-achievable) treatment plan using recursively trialand-error weights for the organs of interest, we go a new way trying to avoid a priori weight choices and consider the treatment planning problem as a multiple objective linear programming problem: with each organ of interest, target tissue as well as organs at risk, we associate an objective function measuring the maximal deviation from the prescribed doses.

We build up a data base of relatively few efficient solutions representing and approximating the variety of Pareto solutions of the multiple objective linear programming problem. This data base can be easily scanned by physicians looking for an adequate treatment plan with the aid of an appropriate online tool.

(14 S., 1999)

\section{C. Lang, J. Ohser, R. Hilfer}

\section{On the Analysis of Spatial Binary Images}

This paper deals with the characterization of microscopically heterogeneous, but macroscopically homogeneous spatial structures. A new method is presented which is strictly based on integral-geometric formulae such as Crofton's intersection formulae and Hadwiger's recursive definition of the Euler number. The corresponding algorithms have clear advantages over other techniques. As an example of application we consider the analysis of spatial digital images produced by means of Computer Assisted Tomography.

(20 S., 1999)

\section{M. Junk}

\section{On the Construction of Discrete Equilibrium} Distributions for Kinetic Schemes

A general approach to the construction of discrete equilibrium distributions is presented. Such distribution func tions can be used to set up Kinetic Schemes as well as Lattice Boltzmann methods. The general principles are also applied to the construction of Chapman Enskog distributions which are used in Kinetic Schemes for com- pressible Navier-Stokes equations. (24 S., 1999)

\section{M. Junk, S. V. Raghurame Rao}

\section{A new discrete velocity method for Navier- Stokes equations}

The relation between the Lattice Boltzmann Method, which has recently become popular, and the Kinetic Schemes, which are routinely used in Computational Fluid Dynamics, is explored. A new discrete velocity mode for the numerical solution of Navier-Stokes equations for incompressible fluid flow is presented by combining both the approaches. The new scheme can be interpreted as pseudo-compressibility method and, for a particular choice of parameters, this interpretation carries over to the Lattice Boltzmann Method. (20 S., 1999)

\section{H. Neunzert}

\section{Mathematics as a Key to Key Technologies}

The main part of this paper will consist of examples, how mathematics really helps to solve industrial problems; these examples are taken from our Institute for Industria Mathematics, from research in the Technomathematics group at my university, but also from ECMI groups and a company called TecMath, which originated 10 years ago from my university group and has already a very successful history.

39 S. (vier PDF-Files), 1999)

\section{J. Ohser, K. Sandau}

\section{Considerations about the Estimation of the} Size Distribution in Wicksell's Corpuscle Problem

Wicksell's corpuscle problem deals with the estimation of the size distribution of a population of particles, all having the same shape, using a lower dimensional sampling probe. This problem was originary formulated for particle systems occurring in life sciences but its solution is of actual and increasing interest in materials science. From a mathematical point of view, Wicksell's problem is an inverse problem where the interesting size distribution is the unknown part of a Volterra equation. The problem is often regarded ill-posed, because the structure of the integrand implies unstable numerical solutions. The accuracy of the numerical solutions is considered here using the condition number, which allows to compare different numerical methods with different (equidistant) class sizes and which indicates, as one result, that a finite section thickness of the probe reduces the numerical problems. Furthermore, the relative error of estimation is computed which can be split into two parts. One part consists of the relative discretization error that increases for increasing class size, and the second part is related to the relative statistical error which increases with decreasing class size. For both parts, upper bounds can be given and the sum of them indicates an optimal class width depending on some specific constants.

(18 S., 1999) 
18. E. Carrizosa, H. W. Hamacher, R. Klein, S. Nickel

\section{Solving nonconvex planar location problems by finite dominating sets}

It is well-known that some of the classical location problems with polyhedral gauges can be solved in polynomial time by finding a finite dominating set, i. e. a finite set of candidates guaranteed to contain at least one optimal location.

In this paper it is first established that this result holds for a much larger class of problems than currently considered in the literature. The model for which this result can be proven includes, for instance, location problems with attraction and repulsion, and location-allocation problems. Next, it is shown that the approximation of general gauges by polyhedral ones in the objective function of our general model can be analyzed with regard to the subsequent error in the optimal objective value. For the approximation problem two different approaches are described, the sandwich procedure and the greedy algorithm. Both of these approaches lead - for fixed epsilon - to polynomial approximation algorithms with accuracy epsilon for solving the general model considered in this paper. Keywords:

Continuous Location, Polyhedral Gauges, Finite Dominating Sets, Approximation, Sandwich Algorithm, Greedy Algorithm

(19 S., 2000)

\section{A. Becker}

\section{A Review on Image Distortion Measures}

Within this paper we review image distortion measures. A distortion measure is a criterion that assigns a "quality number" to an image. We distinguish between mathematical distortion measures and those distortion measures in-cooperating a priori knowledge about the imaging devices (e. g. satellite images), image processing algorithms or the human physiology. We will consider representative examples of different kinds of distortion measures and are going to discuss them. Keywords:

Distortion measure, human visual system

(26 S., 2000)

\section{H. W. Hamacher, M. Labbé, S. Nickel T. Sonneborn}

\section{Polyhedral Properties of the Uncapacitated Multiple Allocation Hub Location Problem}

\section{We examine the feasibility polyhedron of the uncapaci-} tated hub location problem (UHL) with multiple allocation, which has applications in the fields of air passenger and cargo transportation, telecommunication and posta delivery services. In particular we determine the dimension and derive some classes of facets of this polyhedron. We develop some general rules about lifting facets from the uncapacitated facility location (UFL) for UHL and projecting facets from UHL to UFL. By applying these rules we get a new class of facets for UHL which dominates the inequalities in the original formulation. Thus we get a new formulation of UHL whose constraints are all facetdefining. We show its superior computational performance by benchmarking it on a well known data set. Keywords:

integer programming, hub location, facility location, valid inequalities, facets, branch and cut

(21 S., 2000)

\section{H. W. Hamacher, A. Schöbel \\ Design of Zone Tariff Systems in Public Transportation}

Given a public transportation system represented by its stops and direct connections between stops, we consider two problems dealing with the prices for the customers: The fare problem in which subsets of stops are already aggregated to zones and "good" tariffs have to be found in the existing zone system. Closed form solutions for the fare problem are presented for three objective functions. In the zone problem the design of the zones is part of the problem. This problem is NP hard and we therefore propose three heuristics which prove to be very successful in the redesign of one of Germany's transportation systems. (30 S., 2001)

\section{D. Hietel, M. Junk, R. Keck, D. Teleaga: \\ The Finite-Volume-Particle Method for Conservation Laws}

In the Finite-Volume-Particle Method (FVPM), the weak formulation of a hyperbolic conservation law is discretized by restricting it to a discrete set of test functions. In contrast to the usual Finite-Volume approach, the test functions are not taken as characteristic functions of the control volumes in a spatial grid, but are chosen from a partition of unity with smooth and overlapping partition functions (the particles), which can even move along prescribed velocity fields. The information exchange between particles is based on standard numerical flux functions. Geometrical information, similar to the surface area of the cell faces in the Finite-Volume Method and the corresponding normal directions are given as integral quantities of the partition functions.

After a brief derivation of the Finite-Volume-Particle Method, this work focuses on the role of the geometric coefficients in the scheme.

(16 S., 2001)

23. T. Bender, H. Hennes, J. Kalcsics,

\section{T. Melo, S. Nickel}

\section{Location Software and Interface with GIS and Supply Chain Management}

The objective of this paper is to bridge the gap between location theory and practice. To meet this objective focus is given to the development of software capable of addressing the different needs of a wide group of users. There is a very active community on location theory encompassing many research fields such as operations research, computer science, mathematics, engineering, geography, economics and marketing. As a result, people working on facility location problems have a very diverse background and also different needs regarding the software to solve these problems. For those interested in non-commercial applications (e. g. students and researchers), the library of location algorithms (LoLA can be of considerable assistance. LoLA contains a collection of efficient algorithms for solving planar, network and discrete facility location problems. In this paper, a detailed description of the functionality of LoLA is presented. In the fields of geography and marketing, for instance, solving facility location problems requires using large amounts of demographic data. Hence, members of these groups (e. g. urban planners and sales managers) often work with geographical information too s. To address the specific needs of these users, LoLA was inked to a geo- graphical information system (GIS) and the details of the combined functionality are described in the paper. Finally, there is a wide group of practitioners who need to solve large problems and require special purpose software with a good data interface. Many of such users can be found, for example, in the area of supply chain management (SCM). Logistics activities involved in strategic SCM include, among others, facility location planning. In this paper, the development of a commercial location software tool is also described. The too is embedded in the Advanced Planner and Optimizer SCM software developed by SAP AG, Walldorf, Germany. The paper ends with some conclusions and an outlook to future activities.

Keywords:

facility location, software development, geographical information systems, supply chain management. (48 S., 2001)

\section{H. W. Hamacher, S. A. Tjandra Mathematical Modelling of Evacuation Problems: A State of Art}

This paper details models and algorithms which can be applied to evacuation problems. While it concentrates on building evacuation many of the results are applicable also to regional evacuation. All models consider the time as main parameter, where the travel time between components of the building is part of the input and the overall evacuation time is the output. The paper distinguishes between macroscopic and microscopic evacuation models both of which are able to capture the evacuees' movement over time.

Macroscopic models are mainly used to produce good lower bounds for the evacuation time and do not consider any individual behavior during the emergency situation. These bounds can be used to analyze existing buildings or help in the design phase of planning a building. Macroscopic approaches which are based on dynamic network flow models (minimum cost dynamic flow, maximum dynamic flow, universal maximum flow, quickest path and quickest flow) are described. A special feature of the presented approach is the fact, that travel times of evacuees are not restricted to be constant, but may be density dependent. Using multicriteria optimization priority regions and blockage due to fire or smoke may be considered. It is shown how the modelling can be done using time parameter either as discrete or continuous parameter.

Microscopic models are able to model the individual evacuee's characteristics and the interaction among evacuees which influence their movement. Due to the corresponding huge amount of data one uses simulation approaches. Some probabilistic laws for individual evacuee's movement are presented. Moreover ideas to model the evacuee's movement using cellular automata (CA) and resulting software are presented.

In this paper we will focus on macroscopic models and only summarize some of the results of the microscopic approach. While most of the results are applicable to general evacuation situations, we concentrate on building evacuation.

(44 S., 2001) 
25. J. Kuhnert, S. Tiwari

\section{Grid free method for solving the Poisson equation}

A Grid free method for solving the Poisson equation is presented. This is an iterative method. The method is based on the weighted least squares approximation in which the Poisson equation is enforced to be satisfied in every iterations. The boundary conditions can also be enforced in the iteration process. This is a local approximation procedure. The Dirichlet, Neumann and mixed boundary value problems on a unit square are presented and the analytical solutions are compared with the exact solutions. Both solutions matched perfectly. Keywords:

Poisson equation, Least squares method, Grid free method (19 S., 2001)

\section{T. Götz, H. Rave, D. Reinel-Bitzer,}

K. Steiner, H. Tiemeier

\section{Simulation of the fiber spinning process}

To simulate the influence of process parameters to the melt spinning process a fiber model is used and coupled with CFD calculations of the quench air flow. In the fiber model energy, momentum and mass balance are solved for the polymer mass flow. To calculate the quench air the Lattice Boltzmann method is used. Simulations and experiments for different process parameters and hole configurations are compared and show a good agreement.

Keywords:

Melt spinning, fiber model, Lattice Boltzmann, CFD (19 S., 2001)

\section{A. Zemitis}

\section{On interaction of a liquid film with an obstacle}

In this paper mathematical models for liquid films generated by impinging jets are discussed. Attention is stressed to the interaction of the liquid film with some obstacle.

S. G. Taylor [Proc. R. Soc. London Ser. A 253, 313 (1959)] found that the liquid film generated by impinging jets is very sensitive to properties of the wire which was used as an obstacle. The aim of this presentation is to propose a modification of the Taylor's model, which allows to simulate the film shape in cases, when the angle between jets is different from $180^{\circ}$. Numerical results obtained by discussed models give two different shapes of the liquid film similar as in Taylors experiments. These two shapes depend on the regime: either droplets are produced close to the obstacle or not. The difference between two regimes becomes larger if the angle between jets decreases. Existence of such two regimes can be very essential for some applications of impinging jets, if the generated liquid film can have a contact with obstacles. Keywords:

impinging jets, liquid film, models, numerical solution, shape

(22 S., 2001)
28. I. Ginzburg, K. Steiner

Free surface lattice-Boltzmann method to model the filling of expanding cavities by Bingham Fluids

The filling process of viscoplastic metal alloys and plastics in expanding cavities is modelled using the lattice Boltzmann method in two and three dimensions. These models combine the regularized Bingham model for viscoplastic with a free-interface algorithm. The latter is based on a modified immiscible lattice Boltzmann model in which one species is the fluid and the other one is considered as vacuum. The boundary conditions at the curved liquid-vacuum interface are met without any geometrical front reconstruction from a first-order ChapmanEnskog expansion. The numerical results obtained with these models are found in good agreement with available theoretical and numerical analysis.

Keywords:

Generalized LBE, free-surface phenomena, interface boundary conditions, filling processes, Bingham viscoplastic model, regularized models

(22 S., 2001)

\section{H. Neunzert}

\section{"Denn nichts ist für den Menschen als}

Menschen etwas wert, was er nicht mit Leidenschaft tun kann"

Vortrag anlässlich der Verleihung des Akademiepreises des Landes Rheinland-Pfalz am 21.11.2001

Was macht einen guten Hochschullehrer aus? Auf diese Frage gibt es sicher viele verschiedene, fachbezogene Antworten, aber auch ein paar allgemeine Gesichtspunkte: es bedarf der »Leidenschaft« für die Forschung (Max Weber), aus der dann auch die Begeisterung für die Lehre erwächst. Forschung und Lehre gehören zusammen, um die Wissenschaft als lebendiges Tun vermitteln zu können. Der Vortrag gibt Beispiele dafür, wie in angewandter Mathematik Forschungsaufgaben aus praktischen Alltagsproblemstellungen erwachsen, die in die Lehre auf verschiedenen Stufen (Gymnasium bis Graduiertenkolleg) einfließen; er leitet damit auch zu einem aktuellen Forschungsgebiet, der Mehrskalenanalyse mit ihren vielfältigen Anwendungen in Bildverarbeitung, Materialentwicklung und Strömungsmechanik über, was aber nur kurz gestreift wird. Mathematik erscheint hier als eine moderne Schlüsseltechnologie, die aber auch enge Beziehungen zu den Geistes- und Sozialwissenschaften hat

Keywords:

Lehre, Forschung, angewandte Mathematik, Mehrskalenanalyse, Strömungsmechanik

(18 S., 2001)

\section{J. Kuhnert, S. Tiwari}

Finite pointset method based on the projection method for simulations of the incompressible Navier-Stokes equations

A Lagrangian particle scheme is applied to the projection method for the incompressible Navier-Stokes equations. The approximation of spatial derivatives is obtained by the weighted least squares method. The pressure Poisson equation is solved by a local iterative procedure with the help of the least squares method. Numerical tests are performed for two dimensional cases. The Couette flow, Poiseuelle flow, decaying shear flow and the driven cavity flow are presented. The numerical solutions are obtained for stationary as well as instationary cases and are compared with the analytical solutions for channel flows. Finally, the driven cavity in a unit square is considered and the stationary solution obtained from this scheme is compared with that from the finite element method. Keywords:

Incompressible Navier-Stokes equations, Meshfree method, Projection method, Particle scheme, Least squares approximation AMS subject classification:

76D05, 76M28

(25 S., 2001) 